\title{
Improvements in motor tasks through the use of smartphone technology for individuals with Duchenne muscular dystrophy
}

This article was published in the following Dove Press journal:

Neuropsychiatric Disease and Treatment

18 August 2017

Number of times this article has been viewed

\author{
Camila Miliani Capelini' \\ Talita Dias da Silva ${ }^{2}$ \\ James Tonks ${ }^{3-5}$ \\ Suzanna Watson ${ }^{6}$ \\ Mayra Priscila Boscolo \\ Alvarez' \\ Lilian Del Ciello de \\ Menezes' \\ Francis Meire Favero ${ }^{2}$ \\ Fátima Aparecida \\ Caromano' \\ Thais Massetti' \\ Carlos Bandeira de Mello \\ Monteiro' \\ 'Graduate Program in Rehabilitation \\ Sciences, Faculty of Medicine, \\ University of São Paulo, ${ }^{2}$ Department \\ of Medicine, Paulista School of \\ Medicine, Federal University of São \\ Paulo, São Paulo, SP, Brazil; ${ }^{3}$ University \\ of Exeter Medical School, Exeter, \\ ${ }^{4}$ University of Lincoln, Lincoln, \\ ${ }^{5}$ Haven Clinical Psychology Practice, \\ Cornwall, ${ }^{6}$ The Cambridge Centre \\ for Paediatric Neuropsychologicial \\ Rehabilitation, Cambridge, UK
}

Correspondence: Talita Dias da Silva Federal University of São Paulo, Paulista School of Medicine, Rua Sena Madureira, 1500, Vila Clementino, São Paulo, SP 0402I-00I, Brazil Email ft.talitadias@gmail.com
Background: In individuals severely affected with Duchenne muscular dystrophy (DMD), virtual reality has recently been used as a tool to enhance community interaction. Smartphones offer the exciting potential to improve communication, access, and participation, and present the unique opportunity to directly deliver functionality to people with disabilities.

Objective: To verify whether individuals with DMD improve their motor performance when undertaking a visual motor task using a smartphone game.

Patients and methods: Fifty individuals with DMD and 50 healthy, typically developing (TD) controls, aged 10-34 years participated in the study. The functional characterization of the sample was determined through Vignos, Egen Klassifikation, and the Motor Function Measure scales. To complete the task, individuals moved a virtual ball around a virtual maze and the time in seconds was measured after every attempt in order to analyze improvement of performance after the practice trials. Motor performance (time to finish each maze) was measured in phases of acquisition, short-term retention, and transfer.

Results: Use of the smartphone maze game promoted improvement in performance during acquisition in both groups, which remained in the retention phase. At the transfer phases, with alternative maze tasks, the performance in DMD group was similar to the performance of TD group, with the exception of the transfer to the contralateral hand (nondominant). However, the group with DMD demonstrated longer movement time at all stages of learning, compared with the TD group.

Conclusion: The practice of a visual motor task delivered via smartphone game promoted an improvement in performance with similar patterns of learning in both groups. Performance can be influenced by task difficulty, and for people with DMD, motor deficits are responsible for the lower speed of execution. This study indicates that individuals with DMD showed improved performance in a short-term motor learning protocol using a smartphone. We advocate that this technology could be used to promote function in this population.

Keywords: motor skills, physical therapy, cell phone, virtual reality exposure therapy, transfer of learning

\section{Introduction}

Duchenne muscular dystrophy (DMD) is a genetic disease resulting from the mutation of the $X p 21$ gene, which causes lack of the protein dystrophin in the muscle fiber membrane and generates progressive and irreversible weakness of skeletal, cardiac, and respiratory muscles. ${ }^{1-3}$ It is gender specific in adversely affecting only males, and its incidence is $\sim 1$ in 3,500 live male births. ${ }^{4}$ 
Males affected by DMD have severe motor limitations and physical disabilities that lead to wheelchair dependency and the need for assistance in carrying out simple day-to-day activities. ${ }^{5,6}$ The progression of these limitations develops from the lower to upper body segments and in a proximal to distal direction. ${ }^{7}$

A recent and rapidly accruing body of research has considered motor skill tasks for people with DMD. Studies include those designed to improve walking in children with $\mathrm{DMD},{ }^{8,9}$ exercise effects in muscle disuse and functionality, ${ }^{10}$ quality of life, ${ }^{11}$ clinical evaluations of upper limb motor function, ${ }^{12}$ gross motor function and functional disability in mobility, self-care, and social function. ${ }^{13,14}$ There has been a paucity of studies focusing directly upon motor skill tasks to enhance upper limb function in DMD.

This is surprising given that Wagner et $\mathrm{a} \mathrm{l}^{15}$ emphasize that most adults with DMD have limited motor skills, but some muscles of the upper limbs, especially the finger flexors, can be preserved to maintain important skills such as controlling a joystick on a wheelchair, playing video games, and using a computer. Thus, with the continuous improvement in life expectancy through medical advances, ${ }^{16}$ upper limb function deserves specific attention in rehabilitation and research programs in order to prolong independence and quality of life.

For the people severely affected with DMD, technological developments with computers and virtual reality (VR) offer potential for previously unavailable activities that confer greater opportunities to interact with the community. Hashimoto et al, ${ }^{17}$ for example, used a VR task through a brain-computer interface device in order to facilitate interaction between an individual with severe impairments caused by muscular dystrophy and other people. Their findings illustrate how VR can be beneficial to people with motor limitations: people with DMD were able to communicate in a virtual world in the same way as healthy individuals when using the correct equipment for their needs.

In subsequent work, Burgstahler et al ${ }^{18}$ discuss how touch screen devices enable greater functionality for individuals with muscular dystrophy. The authors consider that smartphones and computers enable people with disabilities to maximize independence, productivity, and participation in academic programs, jobs, recreational activities, and other activities. ${ }^{18}$ Further, smartphones enable improved function for people with DMD through utility features such as size, ease and convenience in transportation, flexibility in use, and utilization of distal body structures.

In considering individuals with DMD and the humantechnology interface, individuals with DMD are likely to have sufficient functional skills of hands for the use of keyboards or standard mouse, as shoulders and elbows are commonly the upper limb areas most affected. ${ }^{19,20}$

This study aimed to explore whether people with DMD will improve in motor performance when using a smartphone game. To answer this question, we set up a motor learning protocol with a smartphone maze task for a group of people with DMD and a control group of people with typical development. According to Souza et al, ${ }^{21}$ maze tasks utilize important aspects of planning, execution, spatial organization, and implicit memory, which involve operation of the intention to move to achieve a goal and planning of this action. Maze tasks have been used in Down Syndrome by Menezes et $\mathrm{al}^{22}$ and Possebom et $\mathrm{al}^{23}$ using smartphone and computer, respectively. In both studies, the maze task was found to be appropriate for the motor capacity of the individuals with Down Syndrome, although performance was reduced compared to age-matched control group. The study sample was able to improve and retain performance on the task. In another study, the maze task was also used successfully in assisting individuals with cerebral palsy. ${ }^{24} \mathrm{We}$, therefore, consider that, the use of a maze task to assess motor learning using a smartphone is potentially viable as a training technique to improve motor performance.

In support of our position, we note that Malheiros et $\mathrm{a}^{25}$ used a maze task to assess motor learning using a computer. The authors evaluated 42 individuals with DMD and stated that practice in a maze computational task promoted improvement in performance, although individuals with DMD had reduced movement response speed, compared to an agematched control group.

In summarizing the above research, we note that individuals with DMD are able to learn and benefit from a maze task using a computer, but reasonable distal upper limb movement is needed in performing such tasks. Studies to date have not utilized a maze task on smartphones in populations with DMD, although the work of Burgstahler et al ${ }^{18}$ provides a clear rationale for the use of such technology. It is also the case that little is known about how well transfer of performance may be influenced by maze design.

In this study, we aimed to investigate whether individuals with DMD are able to improve in motor performance during a maze task on a smartphone. To answer this question, we set up a task that required the individuals with DMD to move the smartphone in order to direct a ball in a three-dimensional (3D) virtual environment along a path to reach the intended target using a short-term motor learning protocol. The aim of the game was to perform the task in the shortest time 
possible. It was hypothesized that 1) both experimental and control groups would present performance improvement after several attempts in the acquisition phase, would be able to retain the performance level acquired in a short-term retention phase, would transfer learned performance with changes and 2) the control group would perform better at all stages of the protocol.

\section{Participants and methods Participants}

The study was conducted at the Brazilian Muscular Dystrophy Association located in São Paulo/SP (experimental group). One hundred males aged 10-34 were evaluated after recruiting them for the study. Of these, 50 individuals with a diagnosis of DMD were selected for the experimental group. Individuals were considered eligible for the DMD group if they had a confirmed diagnosis for DMD by molecular method and/or protein expression in skeletal muscle and there was absence of joint deformities that could impede the execution of movements needed to perform the task, such as contracture with total flexion of wrist and fingers and pronation of the forearm, as well as lack of strength to maintain the head bending over to follow the ball's movement on the smartphone. ${ }^{26}$ Individuals who did not complete or understand the task were excluded. We assessed 50 healthy, typically developing (TD) males who were recruited to the control group, and were matched for age and were without any physical conditions affecting posture and movement (Figure 1). This study was approved by the Ethics Committee of the Faculty of Medicine, University of São Paulo (CAAE: 22768513.2.0000.0065).

Individuals of both DMD and TD groups were divided into two subgroups: Group $1(n=25)$ and Group $2(n=25)$; the design of the study is shown in Figure 1. Group 2 used a maze pattern that was the same pattern used in Group 1, but inverted horizontally and vertically in all phases of the

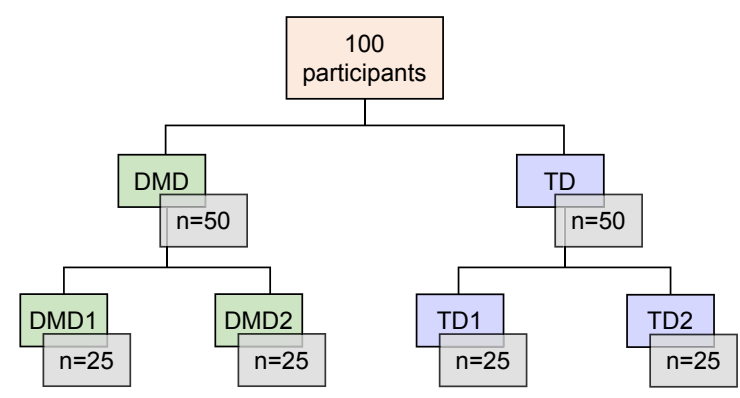

Figure I Study casuistic and composition of groups. Notes: TD: group with TD; DMD: group with DMD.

Abbreviations: DMD, Duchenne muscular dystrophy; TD, typical development. experiment in order to counterbalance presentation effects (essentially, the use of opposite mazes became necessary to ensure that the movement sequence within the maze path did not influence performance). Stoddard and Vaid ${ }^{27}$ also conducted research with maze and proposed different changes in the task of design to see if the movement pattern would not be the factor responsible for the improved performance.

To maintain the characteristics of random sampling, where each individual had an equal chance of participating in the groups, we used simple random probabilistic sampling ${ }^{28}$ by randomly drawing lots with paper.

All individuals of legal age were asked to sign an informed consent form, and minors were given a similar consent agreement, along with the informed consent, which was signed by parents or guardians.

The functional clinical characterization of the individuals with DMD was assessed by the Vignos and Archibald scale, ${ }^{29}$ Egen Klassifikation scale (EK), ${ }^{30}$ and Motor Function Measure (MFM) scale. ${ }^{31}$ These have been validated and can sensitively evaluate functional impairment in neuromuscular diseases. The characterization of individuals is presented in Table 1.

The Vignos is one of the most widely used scales to evaluate the functionality in neuromuscular diseases due to its easiness and simplicity in analysis of muscle performance overall, and EK was specially developed to measure the degree of functional impairment in activities of daily living of patients with DMD. ${ }^{5,30}$

The MFM scale was used to analyze the functions of head, trunk, proximal and distal segments of limbs in static and dynamic evaluations. It is divided into three dimensions: Dimension 1 (D1): standing position and transfers; Dimension 2 (D2): axial and proximal motor function; and

Table I Characterization of the sample (mean \pm SD)

\begin{tabular}{|c|c|c|c|c|c|}
\hline \multirow[t]{2}{*}{ Variables } & \multicolumn{2}{|l|}{ TD } & \multicolumn{2}{|l|}{ DMD } & \multirow[t]{2}{*}{$P$-value } \\
\hline & TD I & TD2 & DMDI & DMD2 & \\
\hline Age (years) & $17.1 \pm 4.3$ & $17.4 \pm 4.4$ & $17.1 \pm 5.3$ & $17.2 \pm 4.7$ & 0.475 \\
\hline MFM-total (\%) & - & - & $49.7 \pm 17.7$ & $47.2 \pm 20.8$ & 0.413 \\
\hline MFM-DI (\%) & - & - & $11.4 \pm 19.9$ & $14.3 \pm 24.2$ & 0.244 \\
\hline MFM-D2 (\%) & - & - & $67.8 \pm 26.7$ & $64.2 \pm 27.4$ & 0.319 \\
\hline MFM-D3 (\%) & - & - & $79.0 \pm 17.9$ & $80.7 \pm 13.2$ & 0.328 \\
\hline EK scale & - & - & $8.6 \pm 6.3$ & $8.6 \pm 6.2$ & 0.435 \\
\hline Vignos & - & - & $6.4 \pm 2.3$ & $6.3 \pm 2.2$ & $0.44 I$ \\
\hline
\end{tabular}

Notes: For differences between DMD groups, Student's $t$-test was performed $(P<0.05)$. TD: group with TD (matched for age with DMD group); DMD: group with DMD. MFM-DI, D2, and D3 indicate scores in the first, second, and third domains of MFM scale, respectively.

Abbreviations: DMD, Duchenne muscular dystrophy; EK, Egen Klassifikation; MFM, Motor Function Measure; SD, standard deviation; TD, typical development. 
Dimension 3 (D3): distal motor function. The lower the score an individual obtains on the scale, the worse is his/her functional picture. ${ }^{31}$

\section{Procedure}

In the data collection phase, a smartphone game called Marble Maze Classic ${ }^{\circledR}$ was used with a Nokia ${ }^{\circledR}$ smartphone, model Nokia 500 (height: $111.3 \mathrm{~mm}$, width: $53.8 \mathrm{~mm}$, thickness: $14.1 \mathrm{~mm}$, weight: $94 \mathrm{~g}$, screen size: $\left.3.2^{\prime \prime}\right)$. The game required individuals to navigate with a virtual ball through a path maze with the goal of reaching a final target in the shortest time possible. The task simulated a wooden table with walls that defined the path of the maze and the virtual ball travels by means of movements performed by the hand holding the smartphone (Figure 2).

Two different customized labyrinths were used in the acquisition phase. The customization of labyrinths was carried out by researchers before the start of the study, by placing walls in order to trace the path to be traveled by the ball.

Each labyrinth path was unique, but there was only one possibility of continuity of the route. Thus, the route to be held by the ball was the same in all trials, at the same phase of protocol. The time taken to move the virtual ball through the maze path until its arrival at the final stop was timed and displayed to the player at the end of the game. Before starting the task, the operation of the game was verbally explained, along with a demonstration made by the examiner. At this point, the need to perform the maze as fast as possible was reinforced.

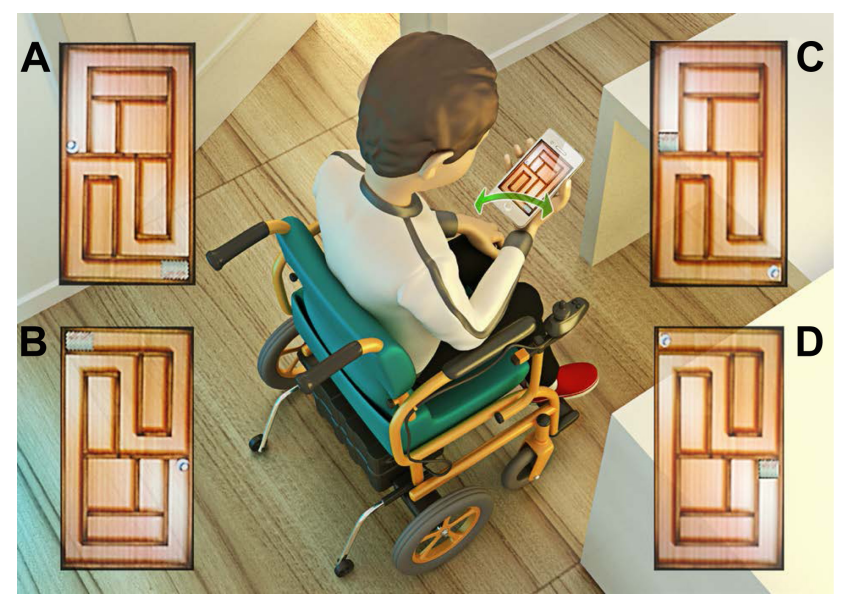

Figure 2 Labyrinths used for the stages of acquisition, short-term retention, and transfer.

Notes: Maze A: used in acquisition and in transfer 2 for groups DMDI and TDI and in transfer I for groups DMD2 and TD2; Maze B: used in acquisition and transfer 2 for groups DMD2 and TD2 and in transfer I for groups DMDI and TDI; Maze C= used in transfer 3 of groups DMDI and TDI; Maze D = used in transfer 3 of groups DMD2 and TD2.

Abbreviations: DMD, Duchenne muscular dystrophy; TD, typical development.

\section{Task}

The task was made up of three phases: the acquisition phase, the short-term retention phase, and the transfer phase. For the learning protocol, individuals performed 30 trials of the task in the acquisition phase with the dominant hand. After the acquisition phase, there was a 5-minute rest, during which the individual had no contact with the task. Then, the individuals were asked to complete five trials in the short-term retention phase using the same maze of acquisition. For the transfer phase, there were 15 trials, divided in three different tasks:

- Transfer 1: used a maze with a path that had a totally opposite layout (inverted vertically and horizontally) to the acquisition maze (five trials);

- Transfer 2: the same maze of acquisition to be carried out with the nondominant hand (five trials);

- Transfer 3: the same maze of acquisition with the start and end points inverted (five trials).

The protocols were the same for both groups; however, the labyrinths used were different (Table 2).

\section{Data analysis}

The dependent variables used were movement time in seconds taken to move through the maze in each trial of the acquisition, short-term retention, and transfer phases, and data were analyzed in blocks, based upon the five trials.

The movement time was defined as the time to finish the maze task. The dependent variables were submitted to a 2 (group: DMD, TD) by 2 (maze type: maze A, maze B) by 2 (block) analysis of variance with repeated measures on the last factor. For the factor block, separate comparisons (contrasts) were made for acquisition (first acquisition block A1 versus final acquisition block A6), short-term retention (A6 versus retention block $R$ ), and transfer ( $R$ versus transfer block T1, R versus transfer block T2, and $\mathrm{R}$ versus transfer block T3). Post hoc comparisons were carried out using Tukey honest significant difference test $(P<0.05)$. The statistical program used was SPSS (Statistical Package for Social Sciences), version 20.0.

\section{Results}

\section{Acquisition}

The pattern of movement time is illustrated in Figure 3. Significant effects were found in the predicted direction for block, $(\mathrm{A} 1-\mathrm{A} 6), F(1,95)=97.2, P<0.001, I^{2}=0.51$, and group, $F(1,95)=64.7, P<0.001, d^{2}=0.41$. These results suggest that the individuals decreased in movement time from A1 $(M=6.3 \mathrm{~s})$ to A6 ( $M=5.2 \mathrm{~s})$. As hypothesized, the DMD group had a much larger movement time $(M=7.0 \mathrm{~s})$ than the TD group $(M=4.5 \mathrm{~s})$. Interactions between block and group were 
Table 2 Experimental design of motor learning protocol

\begin{tabular}{|c|c|c|c|c|c|}
\hline Groups & Acquisition & $\begin{array}{l}\text { Short-term } \\
\text { retention }\end{array}$ & Transfer I & Transfer 2 & Transfer 3 \\
\hline DMDI & Maze A & Maze A & Maze B & Maze A & Maze C \\
\hline \multirow[t]{2}{*}{ TDI } & (30 trials) & (5 trials) & (5 trials) & Nondominant hand (5 trials) & Start/end inverted (5 trials) \\
\hline & Figure $2 \mathrm{~A}$ & Figure $2 \mathrm{~A}$ & Figure $2 \mathrm{~B}$ & Figure $2 \mathrm{~A}$ & Figure $2 \mathrm{C}$ \\
\hline DMD2 & Maze B & Maze B & Maze A & Maze B & Maze D \\
\hline \multirow[t]{2}{*}{ TD2 } & (30 trials) & (5 trials) & (5 trials) & Nondominant hand (5 trials) & Start/end inverted (5 trials) \\
\hline & Figure 2B & Figure 2B & Figure 2A & Figure 2B & Figure 2D \\
\hline
\end{tabular}

Notes: TD: group with TD; DMD: group with DMD.

Abbreviations: DMD, Duchenne muscular dystrophy; TD, typical development.

found, $F(1,95)=6.28, P=0.014, \mathrm{y}^{2}=0.06$. Post hoc analyses failed to identify the differences between block A1 and A6 in the DMD group (mean 7.7 and 6.3, respectively), and the TD group (mean 4.9 and 4.1, respectively).

\section{Short-term retention}

There were no effects or interactions of block for the shortterm retention test. However, a main effect for group was found, $F(1,95)=66.9, P<0.001, \mathrm{y}^{2}=0.41$. This result shows that the movement time was larger $(M=6.3 \mathrm{~s})$ in the DMD group than in the TD group ( $M=4.1 \mathrm{~s})$.

\section{Transfer with opposite maze (TI)}

Significant effects were found for block, $F(1,96)=17.7$, $P<0.001, \mathrm{y}^{2}=0.16$, and group, $F(1,96)=96.2, P<0.001$,

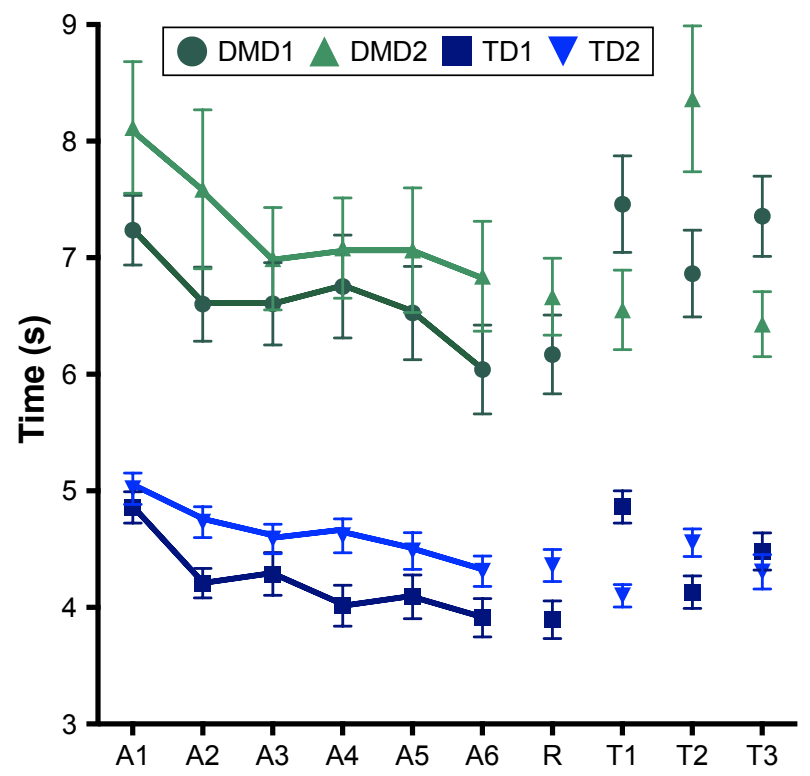

Figure 3 Graphical representation of the means and standard errors of the blocks of trials in TD and DMD groups.

Notes: Each block refers to a set of five trials. AI-A6: blocks of acquisition phase; $\mathrm{R}$ : block of short-term retention test; TI: block of transfer with opposite maze; T2: block of transfer with nondominant hand; T3: block of transfer with exchange start-final. Groups that performed maze A on acquisition: DMDI and TDI. Groups that performed maze B in acquisition: DMD2 and TD2.

Abbreviations: DMD, Duchenne muscular dystrophy; TD, typical development. $d^{2}=0.50$. This result suggests that the individuals increased in movement time from $\mathrm{R}(M=5.2 \mathrm{~s})$ to $\mathrm{T}(M=5.7 \mathrm{~s})$; in addition, the DMD group had a much larger movement time $(M=6.7 \mathrm{~s})$ than the TD group ( $M=4.3 \mathrm{~s})$. Interactions between block by maze type, $F(1,96)=33.1, P<0.001, \eta^{2}=0.26$, were found. Post hoc testing indicated that in maze A, there was a significant increase from block $\mathrm{R}(M=5.0 \mathrm{~s})$ to block $\mathrm{T}(M=6.1 \mathrm{~s})$; for maze $\mathrm{B}$, this difference was not significant $(M=$ from 5.5 to $5.3 \mathrm{~s}$, respectively).

\section{Transfer with nondominant hand (T2)}

Significant effects were found for block, $F(1,96)=23.1$, $P<0.001, \mathrm{n}^{2}=0.19$, and group, $F(1,96)=90.3, P<0.001$, $d^{2}=0.48$. This result suggests that the individuals increased the movement time from $\mathrm{R}(M=5.2 \mathrm{~s})$ to $\mathrm{T}(M=6.0 \mathrm{~s})$; in addition, the DMD group had a much larger movement time $(M=7.0 \mathrm{~s})$ than the TD group $(M=4.2 \mathrm{~s})$. Interactions between block and group, $F(1,96)=6.89, P<0.001$, $\mathrm{y}^{2}=0.19$, were found. Post hoc testing showed that just the DMD group increased in movement time from block $\mathrm{R}(M=6.4 \mathrm{~s})$ to block $\mathrm{T}(M=7.6 \mathrm{~s})$; for the TD group, this difference was not significant $(M=$ from 4.1 to $4.4 \mathrm{~s}$, respectively).

\section{Transfer with exchange start-final (T3)}

Similarly, to transfer with the opposite maze, significant effects were found for block, $F(1,96)=16.5, P<0.001$, $\mathrm{\eta}^{2}=0.15$, and group, $F(1,96)=103.1, P<0.001, \mathrm{\eta}^{2}=0.52$. This result suggests that the individuals increased the movement time from $\mathrm{R}(M=5.2 \mathrm{~s})$ to $\mathrm{T}(M=5.6 \mathrm{~s})$; in addition, the DMD group had a much larger movement time $(M=6.6 \mathrm{~s})$ than the TD group $(M=4.2 \mathrm{~s})$. Interactions between block by maze type, $F(1,96)=30.5, P<0.001, \mathrm{y}^{2}=0.24$, were found. Post hoc test showed that just in maze $\mathrm{A}$, there was a significant increase from block $\mathrm{R}(M=5.0 \mathrm{~s})$ to block T3 $(M=5.9 \mathrm{~s})$; for maze $\mathrm{B}$, this difference was not significant ( $M=$ from 5.5 to $5.3 \mathrm{~s}$, respectively). 


\section{Regression analysis}

In order to determine which factors influenced the degree of learning during practice for the DMD group, a regression analysis was performed between the improvement of movement time from the first to the last practice block $(\Delta)$ and age, MFM-total, MFM-D1, MFM-D2, MFM-D3, EK, Vignos, and block A1. Regression analysis revealed a significant finding, $F(8,46)=3.27, P=0.006, r^{2}=0.41$, resulting in the following equation: improvement $=0.168 \times$ MFM-D1 and $0.401 \times \mathrm{A} 1$. In other words, the score in the first domain of the MFM scale and the movement time in the first practice block predicted the degree of learning.

\section{Discussion}

In this study, we found that, through maze game practice on a smartphone, there was improved performance on the visual motor task of moving a virtual ball around a virtual maze. This was evidenced by the decrease in the movement time from the first to the last block in the acquisition phase and the maintenance of performance at the retention phase in all groups.

While considering the performance of individuals with DMD in undertaking the basic task, we found that they were able to improve their performance with training and adapt to the task, that is, they improved more in the first 15 attempts (blocks A1-A3; Figure 2) and after that, they maintained the good performance acquired, with a little improvement. Regression analysis indicated that the first dimension of the MFM scale (which assessed the standing position and transfers) was positively associated with improvement in performance on the smartphone (ie, higher difference between the first - A1 and the last block of acquisition - A6). This result suggests that the less affected the function of trunk for transfers, the greater the improvement in performance. In addition, the significant finding that $\mathrm{A} 1$ positively influenced performance between A1 and A4 suggests that more improvement is possible when the baseline time to finish the task was quicker. On one hand, this indicates that better functionality leads to better performance improvement. ${ }^{25}$

More generally, the results illustrate that despite physical disabilities, people with DMD are capable of utilizing a smartphone and can benefit from such activity to improve motor performance. Our view is consistent with a previous report by Jover et $\mathrm{al}^{7}$ who ascribe to the view that smartphones are likely to convey increasing adaptive and motor benefits with technological development for disabled populations.

Studies involving motor learning in DMD are recent and have sought to understand how the learning process occurs in these people. In a recent review of the literature, ${ }^{32}$ results which demonstrate that people with DMD maintain motor learning patterns even with the progression of the disease were found; however, other variables are likely to be partial in understanding the resilience of motor learning patterns. Such variables include cognitive ability, deterioration of the motion perception process, and movement impairment in motor learning for people with $\mathrm{DMD}$, for example.

Performance improvement in all groups in the acquisition and retention phases and the similar pattern in performance between the DMD and TD groups were interesting observations. To this extent, differences observed from the group comparisons (TD compared to DMD) are more effectively explicated as a result of shorter movement time in all phases of the protocol for the TD group. Other studies of motor learning in DMD are consistent with the finding that people with typical development have better performance compared to people with DMD. ${ }^{32,33}$ Studies with this population have concluded that this is likely related to muscle dysfunctions that affect motor performance. ${ }^{5,7}$

Recent studies on dexterity and speed in manual tasks with populations affected by neurologic diseases indicate that directly proportional relationships exist between motor control, manual dexterity movement speed, and muscle strength DMD. ${ }^{34-36}$

While considering motor performance in people with DMD, there is a positive correlation between muscle weakness and loss of function in this group, which causes an increase in the time to perform the motor skills. ${ }^{8,37}$ In DMD, this may occur through reduced nervous conduction velocity of the muscle fiber, ${ }^{38,39}$ which leads to a delay in the propagation of excitation, by delaying muscle fiber contraction time. ${ }^{40}$ In addition, there is evidence of fine motor impairment in these individuals, ${ }^{41}$ particularly in relation to the number of skeletal muscle fibers (type II) used in specialized contraction and rapid movement, these fibers are severely affected in DMD. ${ }^{42}$

Bartels et $\mathrm{al}^{20}$ report on the relationship between age and the distal upper limb motor function in DMD, predominantly between 20 and 30 years of age. With advancing age, the global motor function is severely impaired and great variability can be found in the distal motor function, muscle strength, and range of motion of the upper limbs. Mattar and Sobreira ${ }^{43}$ indicate that the strength of the hands in individuals with DMD decreases with age and is significantly different compared to the control groups. Despite such age-associated effects, in our study, regression analyses indicated that this relationship had no apparent effect on performance of the motor response. Further, while exploring the relationship 
between motor function and performance on the task, we similarly found no relationship between the functionality of the hands (Dimension D3 of MFM) and performance. On this basis, we can speculate that hand and wrist movements are still functional with a score of $80 \%$ on average (MFM-D3). Probably because they needed to use distal movements (wrist and/or fingers) to complete the task as an inclusion factor, the severely affected patients were not included in this study.

However, we do report a positive relationship between the Dimension D1 of the MFM, referring to the activities of transfer and postural transitions and improved performance. This indicates that the task executed on the smartphone required a function of the muscles of the body responsible for standing position and transfer, which interfered with the performance of people with DMD. Here, we are of the preliminary opinion that standing and transfers function, mainly, the capacity to stabilize the trunk, was not only responsible for enabling the upper member supports, but also essential in moving the cell, allowing the head to directly look at the screen. Thus, weaker trunk muscle function generates a worse performance in mobile handling. We speculate that this might also account for the poorer performance of the DMD group.

Aside from muscle disorders, neurologic deficiencies and neurochemical function could also influence changes in movement control in DMD. 2,6,44,45 Gao et $\mathrm{al}^{46}$ and Cyrulnik et al, ${ }^{45}$ for example, report that the absence of dystrophin in the cerebellum, which is involved in controlling the speed and accuracy of movements, based upon discriminatory and sensory information is a factor. Winstein et $\mathrm{al}^{47}$ and Witney et $\mathrm{al}^{48}$ have focused instead more exclusively upon the motor cortex, noting that the motor cortex is essential in agile and precise manipulation of objects. In support, there are reports that individuals with DMD present reduced motor cortex excitability and impaired bilateral manual dexterity. ${ }^{49}$

Regarding learned-transfer results, we observed that the design of the maze influenced the performance for the TD and DMD groups. In the transfer test for the opposite maze (T1) and for transfer with exchange start-final (T3), the groups that performed maze A on acquisition (DMD1 and TD1) were unable to transfer performance. However, the groups that performed maze B (DMD2 and TD2) in acquisition were able to transfer. Thus, the groups that performed the maze $B$ transferred to maze $A$, but the groups that practiced maze $A$ did not transfer to maze $B$. This indicates that maze $B$ is probably more difficult.

We can speculate that the movement pattern helps or hinders the performance of the proposed task function in both the DMD and TD groups. In maze B, the part of the path in which the ball should move from bottom to top (third movement) was performed with supination of the forearm, wrist extension and ulnar deviation, which characterizes a pattern of movement more difficult compared to maze A, in which the ball should move from top to bottom (third movement), which demands pronation of the forearm, wrist flexion with ulnar deviation - a more physiologic movement (Li et $\mathrm{al}^{50}$ and O'Sullivan and Gallwey ${ }^{51}$ ). In the case of individuals with DMD, another factor in the difficulty of performing the maze is the presence of muscular contractures, especially in the upper limbs, as this compromises the supination movements of the forearm, as well as the movements of wrist extension..$^{52,53}$

\section{Limitations}

The current study has some limitations. The mazes used were relatively simple and because correlations were not undertaken between the tasks and existing neuropsychological measures, we are uncertain of the extent of cognitive demand. The range of movement was not measured to better characterize the extent of joint deformities. In future work, it would be useful to screen the samples for neurocognitive function and the range of movement of the upper limb to group them accordingly. We also note that the range of executive functions that are involved in maze tasks could also be an area where further understanding is needed.

\section{Conclusion}

We conclude that the practice of a visual motor task in mobile game promoted improvement in performance during the acquisition of the game in groups with DMD and TD Furthermore, this improvement remained in the phases of retention and transfer, showing similar learning patterns for both groups.

For people with DMD, at all stages of the experiment, the time of movement was greater compared to the TD group, since the motor deficits are responsible for the slower speed of the task. However, this does not appear to represent an impediment to the use of smartphones to support the use of upper limb movement for this population of young people.

\section{Acknowledgment}

This study received financial support from the FAPESP (Fundação de Amparo à Pesquisa do Estado de São Paulo, process number 2012/16970-6).

\section{Disclosure}

The authors report no conflicts of interest in this work. 


\section{References}

1. Hoffman EP, Brown RH Jr, Kunkel LM. Dystrophin: the protein product of the Duchenne muscular dystrophy locus. Cell. 1987;51(6): 919-928.

2. Mehler MF. Brain dystrophin, neurogenetics and mental retardation. Brain Res Brain Res Rev. 2000;32(1):277-307.

3. Kohler M, Clarenbach CF, Bahler C, Brack T, Russi EW, Bloch KE. Disability and survival in Duchenne muscular dystrophy. J Neurol Neurosurg Psychiatry. 2009;80(3):320-325.

4. Flanigan KM. Duchenne and Becker muscular dystrophies. Neurol Clin. 2014;32(3):671-688.

5. Nair KP, Vasanth A, Gourie-Devi M, et al. Disabilities in children with Duchenne muscular dystrophy: a profile. J Rehabil Med. 2001;33(4): 147-149.

6. Uchikawa K, Liu M, Hanayama K, Tsuji T, Fujiwara T, Chino N. Functional status and muscle strength in people with Duchenne muscular dystrophy living in the community. J Rehabil Med. 2004;36(3): 124-129.

7. Jover M, Schmitz C, Bosdure E, Chabrol B, Assaiante C. Anticipatory postural adjustments in a bimanual load-lifting task in children with Duchenne muscular dystrophy. Neurosci Lett. 2006;403(3): 271-275.

8. Sienko Thomas S, Buckon CE, Nicorici A, Bagley A, McDonald CM, Sussman MD. Classification of the gait patterns of boys with Duchenne muscular dystrophy and their relationship to function. J Child Neurol. 2010;25(9):1103-1109.

9. Ganea R, Jeannet PY, Paraschiv-Ionescu A, et al. Gait assessment in children with duchenne muscular dystrophy during long-distance walking. J Child Neurol. 2012;27(1):30-38.

10. Jansen M, de Groot IJ, van Alfen N, Geurts A. Physical training in boys with Duchenne Muscular Dystrophy: the protocol of the No Use is Disuse study. BMC Pediatr. 2010;10:55.

11. Bendixen RM, Lott DJ, Senesac C, Mathur S, Vandenborne K. Participation in daily life activities and its relationship to strength and functional measures in boys with Duchenne muscular dystrophy. Disabil Rehabil. 2014;36(22):1918-1923.

12. Mayhew A, Mazzone ES, Eagle M, et al. Development of the Performance of the Upper Limb module for Duchenne muscular dystrophy. Dev Med Child Neurol. 2013;55(11):1038-1045.

13. Vestergaard P, Glerup H, Steffensen BF, Rejnmark L, Rahbek J, Moseklide L. Fracture risk in patients with muscular dystrophy and spinal muscular atrophy. J Rehabil Med. 2001;33(4):150-155.

14. Visser J. Developmental coordination disorder: a review of research on subtypes and comorbidities. Hum Mov Sci. 2003;22(4-5):479-493.

15. Wagner KR, Lechtzin N, Judge DP. Current treatment of adult Duchenne muscular dystrophy. Biochim Biophys Acta. 2007;1772(2):229-237.

16. Eagle M, Baudouin SV, Chandler C, Giddings DR, Bullock R, Bushby K. Survival in Duchenne muscular dystrophy: improvements in life expectancy since 1967 and the impact of home nocturnal ventilation. Neuromuscul Disord. 2002;12(10):926-929.

17. Hashimoto Y, Ushiba J, Kimura A, Liu M, Tomita Y. Change in brain activity through virtual reality-based brain-machine communication in a chronic tetraplegic subject with muscular dystrophy. BMC Neurosci. 2010;11:117.

18. Burgstahler S, Comden D, Lee S-M, Arnold A, Brown K. Computer and cell phone access for individuals with mobility impairments: an overview and case studies. NeuroRehabilitation. 2011;28(3):183-197.

19. James WV, Orr JF. Upper limb weakness in children with Duchenne muscular dystrophy-a neglected problem. Prosthet Orthot Int. 1984; $8(2): 111-113$.

20. Bartels B, Pangalila RF, Bergen MP, Cobben NA, Stam HJ, Roebroeck ME. Upper limb function in adults with Duchenne muscular dystrophy. J Rehabil Med. 2011;43(9):770-775.

21. Souza D, França F, Campos T. Teste de labirinto: instrumento de análise na aquisição de uma habilidade motora. Braz J Phys Ther. (Impr.). 2006; 10(3):355-360.
22. Menezes LDCd, Gomes KdSC, Massetti T, et al. Motor learning in mobile (cell phone) device in Down syndrome patients-pilot project. MedicalExpress. 2015;2(4):1-5.

23. Possebom WF, Massetti T, da Silva TD, et al. Maze computer performance in Down syndrome. J Human Growth Develop. 2016;26(2): 205-210.

24. Paula JN, Ahn RÉ, Monteiro C, et al. Aprendizagem motora por meio de jogo de telefone celular na Paralisia Cerebral - Estudo piloto. Arquivos Brasileiros de Paralisia Cerebral. 2015;7:26-31.

25. Malheiros SR, da Silva TD, Favero FM, et al. Computer task performance by subjects with Duchenne muscular dystrophy. Neuropsychiatr Dis Treat. 2016;12:41-48.

26. da Silva TD, de Oliveira EP, Balbino GC, et al. Pegboard task in Duchenne muscular dystrophy. HealthMED. 2015;9(9):365.

27. Stoddard J, Vaid J. Asymmetries in intermanual transfer of maze learning in right- and left-handed adults. Neuropsychologia. 1996;34(6): 605-608.

28. Pires NCM, Arantes EC, Silva W, Kato HT. Diferenças e semelhanças nos métodos de amostragem de pesquisas top of mind: um estudo comparativo. Revista Brasileira de Gestão de Negócios. 2006;8(22):37-45.

29. Vignos PJ Jr, Archibald KC. Maintenance of ambulation in childhood muscular dystrophy. J Chronic Dis. 1960;12:273-290.

30. Martinez JA, Brunherotti MA, de Assis MR, Sobreira CF. Validação da escala motora funcional EK para a língua portuguesa [Validation of the EK functional motor scale in the Portuguese language]. Rev Assoc Med Bras (1992). 2006;52(5):347-351. Portuguese.

31. Berard C, Payan C, Hodgkinson I, Fermanian J. A motor function measure for neuromuscular diseases. Construction and validation study. Neuromuscul Disord. 2005;15(7):463-470.

32. Malheiros SRP, Ribeiro DC, Alvarez MPB, et al. Motor learning in duchenne muscular dystrophy, a literature review. Int Arch Med. $2015 ; 8: 217$

33. Nakafuji A, Tsuji K. Learning and transfer in two perceptual-motor skills in Duchenne muscular dystrophy. Percept Mot Skills. 2001; 93(2):339-352.

34. Smith DL, Dainoff MJ, Smith JP. The effect of chiropractic adjustments on movement time: a pilot study using Fitts Law. J Manipulative Physiol Ther. 2006;29(4):257-266.

35. Kinali M, Arechavala-Gomeza V, Feng L, et al. Local restoration of dystrophin expression with the morpholino oligomer AVI-4658 in Duchenne muscular dystrophy: a single-blind, placebo-controlled, dose-escalation, proof-of-concept study. Lancet Neurol. 2009; 8(10):918-928.

36. Bezdicek O, Nikolai T, Hoskovcova M, et al. Grooved pegboard predicates more of cognitive than motor involvement in Parkinson's disease. Assessment. 2014;21(6):723-730.

37. Scott OM, Hyde SA, Goddard C, Dubowitz V. Quantitation of muscle function in children: a prospective study in Duchenne muscular dystrophy. Muscle Nerve. 1982;5(4):291-301.

38. Cruz Martinez A, Lopez Terradas JM. Conduction velocity along muscle fibers in situ in Duchenne muscular dystrophy. Arch Phys Med Rehabil. 1990;71(8):558-561.

39. Al-Ani FS, Hamdan FB, Shaikhly KI. In situ measurements of muscle fiber conduction velocity in Duchenne muscular dystrophy. Saudi Med J. 2001;22(3):259-261.

40. Murakami K, Fujisawa H, Onobe J, Sato Y. Relationship between Muscle Fiber Conduction Velocity and the Force-time Curve during Muscle Twitches. J Phys Ther Sci. 2014;26(4):621-624.

41. Mento G, Tarantino V, Bisiacchi PS. The neuropsychological profile of infantile Duchenne muscular dystrophy. Clin Neuropsychol. 2011; 25(8):1359-1377.

42. Webster C, Silberstein L, Hays AP, Blau HM. Fast muscle fibers are preferentially affected in Duchenne muscular dystrophy. Cell. 1988; 52(4):503-513.

43. Mattar FL, Sobreira C. Hand weakness in Duchenne muscular dystrophy and its relation to physical disability. Neuromuscul Disord. 2008; 18(3):193-198. 
44. Anderson JL, Head SI, Rae C, Morley JW. Brain function in Duchenne muscular dystrophy. Brain. 2002;125(Pt 1):4-13.

45. Cyrulnik SE, Fee RJ, Batchelder A, Kiefel J, Goldstein E, Hinton VJ. Cognitive and adaptive deficits in young children with Duchenne muscular dystrophy (DMD). J Int Neuropsychol Soc. 2008;14(5):853-861.

46. Gao JH, Parsons LM, Bower JM, Xiong J, Li J, Fox PT. Cerebellum implicated in sensory acquisition and discrimination rather than motor control. Science. 1996;272(5261):545-547.

47. Winstein CJ, Grafton ST, Pohl PS. Motor task difficulty and brain activity: investigation of goal-directed reciprocal aiming using positron emission tomography. J Neurophysiol. 1997;77(3):1581-1594.

48. Witney AG, Wing A, Thonnard JL, Smith AM. The cutaneous contribution to adaptive precision grip. Trends Neurosci. 2004;27(10): 637-643.

49. Lee JS, Pfund Z, Juhasz C, et al. Altered regional brain glucose metabolism in Duchenne muscular dystrophy: a pet study. Muscle Nerve. 2002;26(4):506-512.
50. Li ZM, Kuxhaus L, Fisk JA, Christophel TH. Coupling between wrist flexion-extension and radial-ulnar deviation. Clin Biomech (Bristol, Avon). 2005;20(2):177-183.

51. O'Sullivan LW, Gallwey TJ. Forearm torque strengths and discomfort profiles in pronation and supination. Ergonomics. 2005;48(6): 703-721.

52. Skalsky AJ, McDonald CM. Prevention and management of limb contractures in neuromuscular diseases. Phys Med Rehabil Clin N Am 2012;23(3):675-687.

53. Servais L, Deconinck N, Moraux A, et al. Innovative methods to assess upper limb strength and function in non-ambulant Duchenne patients. Neuromuscul Disord. 2013;23(2):139-148.
Neuropsychiatric Disease and Treatment

\section{Publish your work in this journal}

Neuropsychiatric Disease and Treatment is an international, peerreviewed journal of clinical therapeutics and pharmacology focusing on concise rapid reporting of clinical or pre-clinical studies on a range of neuropsychiatric and neurological disorders. This journal is indexed on PubMed Central, the 'PsycINFO' database and CAS,

\section{Dovepress}

and is the official journal of The International Neuropsychiatric Association (INA). The manuscript management system is completely online and includes a very quick and fair peer-review system, which is all easy to use. Visit http://www.dovepress.com/testimonials.php to read real quotes from published authors.

Submit your manuscript here: http://www.dovepress.com/neuropsychiatric-disease-and-treatment-journal 